Gut, 1986, 27, 1330-1337

\title{
T-lymphocyte populations in normal and coeliac small intestinal mucosa defined by monoclonal antibodies
}

\author{
D JENKINS, ANNE GOODALL, AND B B SCOTT \\ From the Departments of Medicine and Pathology, Lincoln County Hospital, Lincoln
}

SUMmaRY Counts of T-lymphocytes within surface and crypt epithelium and lamina propria of normal and coeliac small intestinal mucosa using an immunoperoxidase method are described and related statistically to changes in mucosal structure determined by quantitative histology. The use of multiple pan-T-lymphocyte and subset antibodies allowed comparison of marker patterns in normal and abnormal mucosa. Total numbers of surface epithelial T-lymphocytes and subtypes were not found to be increased in coeliac disease, but there was an increase in density per unit length of epithelium. Distinctive changes in expression of Leu 1, Leu 2, and Leu 5 by the surface epithelial T-lymphocytes suggest that, although their total numbers are not increased, these cells are not passively crowded but have an active role, possibly as committed cytotoxic lymphocytes. Natural killer cells do not appear to be a major population in normal or coeliac small intestinal mucosa.

The mechanism whereby gluten causes mucosal damage in coeliac disease is not known although there is much evidence of altered humoral and cell mediated immunity. Studies using the models of allograft rejection in mice ${ }^{1}$ and the demonstration of cellular sensitisation to gluten fraction III in the peripheral blood of patients with coeliac disease ${ }^{2}$ suggest that cell mediated immunity is important in the pathogenesis of coeliac disease. The presence of serum antibodies to gluten and the demonstration of antigliadin antibody production by cultured coeliac mucosa exposed to gluten ${ }^{3}$ show that there is also a humoral immune response.

In the small intestinal mucosa of untreated coeliac disease there is a marked rise in the number of $\operatorname{IgM}$, $\operatorname{IgA}$ and $\operatorname{IgE}$ producing plasma cells. ${ }^{4}$ Both $\operatorname{IgA}$ and IgE antibody responses are regulated by subsets of T-lymphocytes and therefore T-lymphocytes may have an important role in both humoral and cellmediated immunity in coeliac disease. It has been shown that T-suppressor lymphocyte activity is generated by alpha gliadin exposure in coeliac disease. ${ }^{5}$ Morphological and morphometric studies have focused attention on the changes in number and size of intraepithelial lymphocytes in coeliac

Address for correspondence: Dr B B Scott, Department of Medicine, Lincoln County Hospital, Lincoln LN2 5QY.

Received for publication 14 February 1986. disease and suggested that they might be of particular importance in pathogenesis. ${ }^{6-8}$ These have been shown to consist predominantly of T-lymphocytes carrying the T8 antigen ${ }^{9}{ }^{10}$ which has been used as a marker for the 'suppressor/cytotoxic' subset. It has also been suggested that other cytotoxic effector cells such as killer, or $\mathrm{K}$, cells ${ }^{11}$ and natural killer, or NK, cells may have a role.

Fluorescence microscopic studies have shown similar distributions and ratios of $\mathrm{T} 4$ and $\mathrm{T} 8$ positive T-lymphocytes in the surface epithelium and lamina propria of normal and coeliac small bowel mucosa, ${ }^{9} 10$ and have failed to show any abnormalities in the phenotype of T-lymphocytes which might support a major role in coeliac disease. Although it appeared initially that the T4 positive lymphocytes formed a 'helper/inducer' subset and T8 positive cells a 'suppressor/cytotoxic' subset of T-lymphocytes, more recent studies have shown that such a view conceals a much greater complexity of relationship between phenotype and function, ${ }^{12}$ and that the T4 and T8 molecules may be involved in the recognition of class I and II histocompatibility antigens. ${ }^{13}$ It has also become clear that the different 'pan- $T$ ' antibodies reacting to near $100 \%$ of $\mathrm{T}$-lymphocytes identify several different glycoprotein markers on Tlymphocytes. The $\mathrm{T} 3$ antigen appears to be the most reliable indicator of a functionally mature peripheral T-lymphocyte. Other antibodies may identify other 
cells in addition or fail to identify all functional T-lymphocytes. Quantitative information on the range of normal values for lymphocyte populations, defined by the different pan-T-lymphocyte and subset antibodies, in surface and crypt epithelium and in the lamina propria is not available. Neither have the changes in coeliac disease been described quantitatively. Such information allows the recognition of changes in the marker profile of the lymphocyte populations in disease which might indicate a change in the functional state of the cells that cannot be suspected from a simple qualitative or even semi-quantitative study. It also allows correlation of changes in the populations with alterations in mucosal architecture as an approach to the study of the relationships between these cell populations and mucosal structural change.

We describe the use of an immunoperoxidase method using multiple antibodies of possible pan-Tlymphocyte specificity (anti-Leu 1, anti-Leu 5, anti-Leu 9 and anti-T3) and two T-subset specific antibodies (anti-Leu 2 and anti-Leu 3 which have been regarded as markers for cytotoxic/suppressor and helper/inducer cells respectively) to define the range of cell counts in controls and coeliac patients. The statistical relationships between T-lymphocyte populations and measurements of villous and crypt epithelium and lamina propria are presented. The results of staining with the NK cell markers, antiLeu 7 and anti-Leu 11, are also described.

\section{Methods}

\section{SUBJECTS}

\section{CONTROLS}

Twenty patients undergoing routine small bowel biopsy for the investigation of diarrhoea or anaemia who were found to have no evidence of small bowel disease. There were 10 men. The mean age was 36 years with a range of 14-69 years. For studies of T3 and Leu 5 markers only eight of these controls were used.

\section{COELIAC DISEASE PATIENTS}

Seventeen patients undergoing routine small bowel biopsy for the investigation of suspected small bowel disease. Biopsies from all these patients showed either subtotal villous atrophy or severe partial villous atrophy. They were all subsequently treated with a gluten free diet with marked clinical improvement. All those 12 who have had a further biopsy have shown definite histological improvement. Two patients also had dermatitis herpetiformis. Seven were men. The mean age was 40 years with a range of 8-71 years.
SMALL INTESTINAL BIOPSY

Multiple, usually eight, biopsies were taken from the junction of the second and third parts of duodenum with either FB-13K or FB-24K forceps passed down either an Olympus GIF-Q or GIF-T endoscope. They were immediately orientated on plastic mesh using a stereomicroscope as previously described. ${ }^{14}$ One specimen was then frozen in liquid nitrogen for the study and the rest were fixed in formalin and sent for routine histology.

\section{MONOCLONAL ANTIBODIES}

ANTI-T3

The T3 antigen is the closest approximation to a pan-T cell antigen.

ANTI-LEU 1

Leu 1 is an antigen found on all circulating T-cells and thymocytes.

ANTI-LEU 9

Leu 9 is an antigen found on human T-lymphocytes and null cells.

ANTI-LEU 5

Leu 5 is an antigen associated with the E-rosette receptor.

ANTI-LEU 2

Type 2a. Leu 2 has been regarded as a marker of the cytotoxic/suppressor subset of T-lymphocytes.

ANTI-LEU 3

Type 3a. Leu 3 has been regarded as a marker of the helper/inducer subset of T-lymphocytes.

ANTI-LEU 7

Leu 7 is an antigen found on NK cells.

ANTI-LEU 11

Leu 11 is an antigen found on NK cells.

All antibodies except anti-T3 were obtained from Becton-Dickinson, Sunnyvale, California, USA. Anti-T3 as UCHT1 was donated by Dr P Beverley, ICRF Tumour Immunology Unit, University College Hospital, London.

STAINING TECHNIQUES

The frozen biopsies were embedded in OCT compound (Tissue Tek II, Miles Scientific) and sectioned perpendicularly to the muscularis mucosae using a Reichert Jung Frigocut. Nine consecutive 5 $\mu \mathrm{m}$ sections were taken from each biopsy, orientated consistently on glass microscope slides with two sections to each slide, and air dried. They were then fixed in $100 \%$ ethanol for 10 minutes and air dried again. All the monoclonal antibodies were diluted 1:5 with Tris-buffered saline (TBS), $\mathrm{pH} 7 \cdot 6$ 
for use, and applied directly to the dry sections. The first eight sections were incubated for one hour each with monoclonal antibody, followed by biotinylated antibody to mouse IgG (Vector Laboratories) and a performed biotinylated peroxidase-avidin DH complex (Vectastain, Vector Laboratories), with three five minute washes in TBS between each reagent. All incubations were carried out at room temperature in a humidified chamber. The sections were then incubated for 10 minutes with $0.5 \% 3,3^{\prime}$ diaminobenzidene in TRIS buffer, $\mathrm{pH} 7 \cdot 6$, to which $0.033 \%$ hydrogen peroxide (final concentration) had been added immediately before use, washed three times in TBS, incubated for a further 10 minutes in $1.5 \%$ copper sulphate solution, washed again, counterstained with Mayer's haematoxylin and mounted. The ninth section from each biopsy received no monoclonal antibody in the first step but was otherwise treated similarly throughout in order to identify cells with endogenous peroxidase/ pseudoperoxidase activity.

\section{CELL COUNTING}

Measurements and cell counts on the immunoperoxidase stained sections were carried out using a MOPPET image analyser. An image of the stained sections was projected at standard magnification on to the digitising tablet by a Leitz Neopromar projection microscope. The MOP stylus was fitted with a ball point pen to provide a permanent record of the field examined. Four contiguous, nonoverlapping fields were examined on each section. A field was defined as the area between the mucosal surface and muscularis mucosae bounded by two lines perpendicular to the muscularis mucosae. The following four measurements were made on each field: (1) mucosal length - the length of muscularis mucosae; (2) area of lamina propria; (3) epithelial cell length of both crypts and surface; (4) counts of positively stained lymphocytes in the crypt epithelium, surface or villous epithelium, and lamina propria. A micrometer slide was used to convert area and length measurements on the projected image into absolute measurements. For each section epithelial cell counts were expressed as cells per mm muscularis mucosae and cells per mm epithelial length. Lamina propria cell counts were expressed as cells per mm muscularis mucosae and cells per $\mathrm{mm}^{2}$ of lamina propria area. The same four fields were counted on each section wherever it was possible. All measurements and processing were performed blind on randomised specimens to minimise experimental and observer bias.

\section{STATISTICAL METHODS}

Arithmetic means and standard deviations of numbers of lymphocytes reacting with each antibody were calculated for each site and compared using an unpaired Student's $t$ test.

\section{Results}

The numbers of lymphocytes reacting with each monoclonal antibody at each site are given in Tables 1-5 together with the significance of differences between controls and untreated coeliac patients. In both controls and coeliacs the number of intraepithelial lymphocytes demonstrated by the $\mathrm{T}$ lymphocyte marker, T3, and by Leu 9 staining are equivalent. There is no significant excess for Leu 9

Table 1 Surface epithelial lymphocytes/mm surface epithelium

\begin{tabular}{|c|c|c|c|c|c|c|c|c|c|}
\hline \multirow{2}{*}{$\begin{array}{l}\text { Lymphocyte } \\
\text { marker }\end{array}$} & \multicolumn{4}{|c|}{ Controls } & \multicolumn{5}{|c|}{ Untreated coeliacs } \\
\hline & $n$ & Range & Mean & $S D$ & $n$ & Range & Mean & $S D$ & \\
\hline $\mathrm{T} 3$ & 8 & $14-58$ & 34 & $16 \cdot 3$ & 15 & $45-180$ & 116 & 29.9 & $*$ \\
\hline Leu 9 & 20 & $12-81$ & 35 & $17 \cdot 4$ & 17 & $27-184$ & 102 & $36 \cdot 7$ & * \\
\hline Leu 1 & 20 & $6-53$ & 18 & $11 \cdot 9$ & 17 & $10-133$ & 64 & $36 \cdot 8$ & * \\
\hline Leu 5 & 8 & $12-48$ & 25 & $14 \cdot 3$ & 15 & $33-120$ & 72 & $24 \cdot 7$ & * \\
\hline Leu 2 & 20 & $9-71$ & 28 & $18 \cdot 0$ & 17 & $13-105$ & 68 & $26 \cdot 6$ & * \\
\hline Leu 3 & 20 & $1-14$ & 3 & $2 \cdot 8$ & 17 & $1-20$ & 9 & $4 \cdot 8$ & * \\
\hline T3 - (Leu $2+3)$ & 8 & $-28-20$ & 0 & $14 \cdot 2$ & 15 & $8-60$ & 35 & $21 \cdot 4$ & * \\
\hline T3 - Leu 1 & 8 & $-1-38$ & 16 & $13 \cdot 8$ & 15 & $-9-92$ & 48 & $24 \cdot 4$ & $\ddagger$ \\
\hline T3 - Leu 5 & 8 & $-11-36$ & 9 & $13 \cdot 8$ & 15 & $12-101$ & 44 & $24 \cdot 3$ & $\dagger$ \\
\hline Leu $9-($ Leu $2+3)$ & 20 & $-13-33$ & 5 & $11 \cdot 0$ & 17 & $5-86$ & 26 & $19 \cdot 4$ & $*$ \\
\hline
\end{tabular}


Table 2 Surface epithelial lymphocytes/mm muscular mucosae

\begin{tabular}{|c|c|c|c|c|c|c|c|c|}
\hline \multirow{2}{*}{$\begin{array}{l}\text { Lymphocyte } \\
\text { marker }\end{array}$} & \multicolumn{4}{|c|}{ Controls } & \multicolumn{4}{|c|}{ Untreated coeliacs } \\
\hline & $n$ & Range & Mean & $S D$ & $n$ & Range & Mean & $S D$ \\
\hline T3 & 8 & $47-213$ & 136 & $57 \cdot 5$ & 15 & $39-316$ & 152 & $63 \cdot 2$ \\
\hline Leu 9 & 20 & $52-316$ & 153 & $77 \cdot 3$ & 17 & $49-214$ & 129 & $50 \cdot 9$ \\
\hline Leu 1 & 20 & $16-178$ & 72 & $47 \cdot 1$ & 17 & $14-170$ & 70 & $38 \cdot 1$ \\
\hline Leu 5 & 8 & $49-248$ & 103 & $66 \cdot 8$ & 15 & $26-152$ & 92 & $37 \cdot 4$ \\
\hline Leu 2 & 20 & $27-309$ & 115 & $77 \cdot 8$ & 17 & $6-151$ & 86 & $41 \cdot 3$ \\
\hline Leu 3 & 20 & $3-48$ & 12 & $9 \cdot 8$ & 17 & $4-21$ & 10 & $5 \cdot 4$ \\
\hline T3 - (Leu $2+3)$ & 8 & $-115-74$ & 2 & $55 \cdot 1$ & 15 & $-8-144$ & 50 & $35 \cdot 8$ \\
\hline T3 - Leu 1 & 8 & $31-157$ & 71 & $49 \cdot 5$ & 15 & $2-222$ & 77 & $56 \cdot 5$ \\
\hline T3 - Leu 5 & 8 & $-35-93$ & 34 & $40 \cdot 0$ & 15 & $-1-211$ & 60 & $52 \cdot 7$ \\
\hline Leu $9-($ Leu $2+3)$ & 20 & $-42-123$ & 29 & $42 \cdot 2$ & 17 & $-4-90$ & 33 & $25 \cdot 7$ \\
\hline
\end{tabular}

None of the differences between the coeliacs and controls is statistically significant.

Table 3 Crypt epithelial lymphocytes/mm crypt epithelium

\begin{tabular}{|c|c|c|c|c|c|c|c|c|}
\hline \multirow{2}{*}{$\begin{array}{l}\text { Lymphocyte } \\
\text { marker }\end{array}$} & \multicolumn{4}{|c|}{ Controls } & \multicolumn{4}{|c|}{ Untreated coeliacs } \\
\hline & $n$ & Range & Mean & $S D$ & $n$ & Range & Mean & $S D$ \\
\hline T3 & 8 & $4-26$ & 13 & $7 \cdot 8$ & 15 & $12-47$ & 19 & $10 \cdot 4$ \\
\hline Leu 9 & 20 & $2-22$ & 9 & $5 \cdot 4$ & 17 & $5-33$ & 15 & $8 \cdot 5$ \\
\hline Leu 1 & 20 & $3-23$ & 7 & $5 \cdot 6$ & 17 & $2-26$ & 11 & $10 \cdot 5$ \\
\hline Leu 5 & 8 & $4-19$ & 9 & $4 \cdot 6$ & 15 & $2-29$ & 9 & $7 \cdot 3$ \\
\hline Leu 2 & 20 & $1-20$ & 8 & $5 \cdot 0$ & 17 & $4-22$ & 11 & $5 \cdot 6$ \\
\hline Leu 3 & 20 & $0-6$ & 2 & $1 \cdot 3$ & 17 & $0-7$ & 2 & 1.9 \\
\hline $\mathrm{T} 3-($ Leu $2+3)$ & 8 & $-19-7$ & 0 & $8 \cdot 1$ & 15 & $-4-19$ & 5 & $5 \cdot 8$ \\
\hline T3 - Leu 1 & 8 & $-1-11$ & 5 & $4 \cdot 2$ & 15 & $-5-28$ & 8 & $7 \cdot 7$ \\
\hline T3 - Leu 5 & 8 & $-4-16$ & 4 & $5 \cdot 6$ & 15 & $0-27$ & 10 & $6 \cdot 2$ \\
\hline Leu $9-($ Leu $2+3)$ & 20 & $-8-6$ & -1 & $3 \cdot 7$ & 17 & $-16-15$ & 3 & $7 \cdot 2$ \\
\hline
\end{tabular}

None of the differences between the coeliacs and controls is statistically significant.

Table 4 Crypt epithelial lymphocytes/mm muscularis mucosae

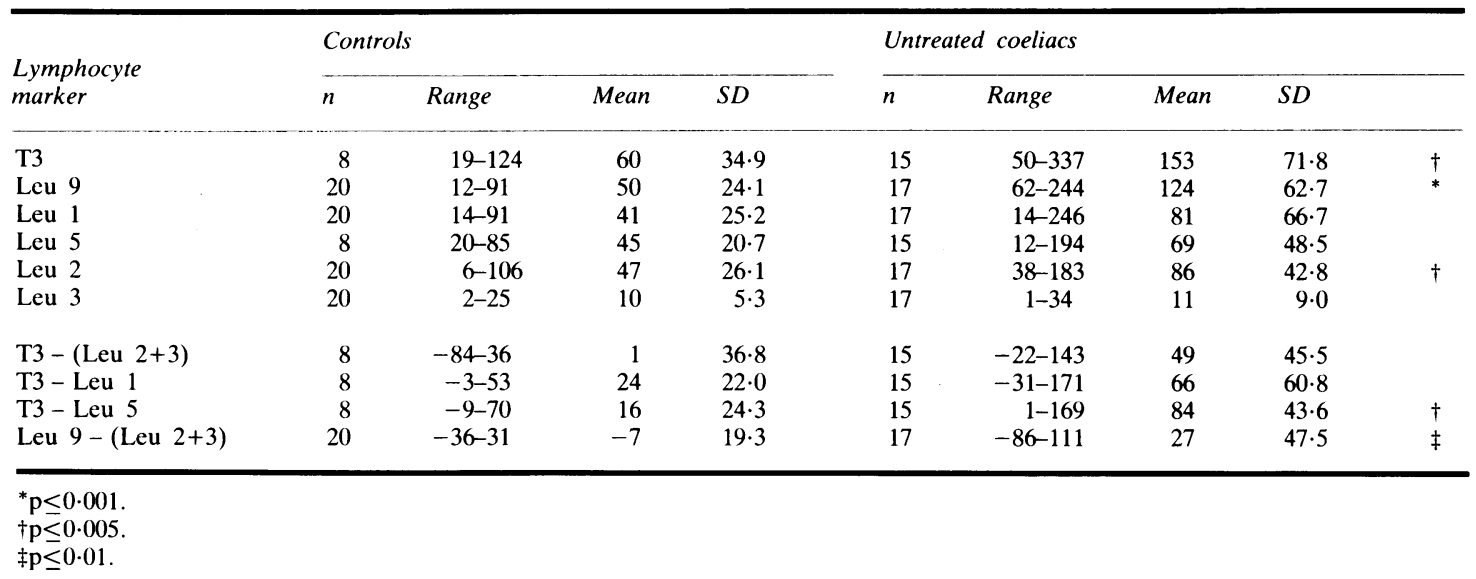


Table 5 Lamina propria lymphocytes $/ \mathrm{mm}$ muscularis mucosae

\begin{tabular}{|c|c|c|c|c|c|c|c|c|}
\hline \multirow{2}{*}{$\begin{array}{l}\text { Lymphocyte } \\
\text { marker }\end{array}$} & \multicolumn{4}{|c|}{ Controls } & \multicolumn{4}{|c|}{ Untreated coeliacs } \\
\hline & $n$ & Range & Mean & $S D$ & $n$ & Range & Mean & $S D$ \\
\hline $\mathrm{T} 3$ & 8 & $124-442$ & 263 & $108 \cdot 5$ & 15 & $123-859$ & 445 & $200 \cdot 3$ \\
\hline Leu 9 & 20 & $68-338$ & 143 & $78 \cdot 7$ & 17 & $91-736$ & 274 & $194 \cdot 6$ \\
\hline Leu 1 & 20 & $104-551$ & 286 & $132 \cdot 3$ & 17 & $102-820$ & 372 & 181.9 \\
\hline Leu 5 & 8 & $125-471$ & 267 & 115.4 & 15 & $60-976$ & 370 & $257 \cdot 3$ \\
\hline Leu 2 & 20 & $26-200$ & 103 & $52 \cdot 5$ & 17 & $37-372$ & 158 & $95 \cdot 3$ \\
\hline Leu 3 & 20 & $63-541$ & 255 & $123 \cdot 6$ & 17 & $121-585$ & 331 & $162 \cdot 0$ \\
\hline T3 - (Leu 2+3) & 8 & $-208-30$ & -64 & $89 \cdot 0$ & 15 & $-390-555$ & -18 & $252 \cdot 7$ \\
\hline T3-Leu 1 & 8 & $-101-128$ & -4 & $69 \cdot 9$ & 15 & $-313-462$ & 91 & $209 \cdot 9$ \\
\hline T3 - Leu 5 & 8 & $-130-82$ & -5 & $69 \cdot 6$ & 15 & $-117-293$ & 75 & $106 \cdot 1$ \\
\hline
\end{tabular}

None of the differences between the coeliacs and controls is statistically significant.

staining. Leu 9 stains both null cells and Tlymphocytes. The fact that the Leu 9 positive cells can be accounted for numerically by $\mathrm{T} 3$ positive $\mathrm{T}$ lymphocytes, together with the presence of only a few cells staining with anti-Leu 7 or anti-Leu 11, suggests that there is a paucity of $\mathrm{NK}$ and $\mathrm{K}$ cells in both normal and coeliac mucosa. In coeliac disease there is a highly significant increase in intraepithelial T3 and Leu 9 positive T-lymphocytes per $\mathrm{mm}$ of surface epithelium compared with controls. The counts of T3 and Leu 9 positive surface epithelial lymphocytes per unit length of underlying muscularis mucosae are, however, similar in controls and coeliacs. In both controls and coeliacs the counts of T3 and Leu 9 positive lymphocytes per $\mathrm{mm}$ of epithelium are higher in the surface epithelium than in the crypt epithelium $(p<0.001$ except for T3 in controls where $p<0 \cdot 01$ ). The counts related to the length of muscularis mucosae are also higher in the surface epithelium than in the crypt epithelium of controls, but in coeliac disease the counts are similar at the two sites. In coeliac disease there is no significant increase in the crypt epithelial T3 and Leu 9 positive lymphocytes per $\mathrm{mm}$ of crypt epithelium, but the counts are increased in coeliacs when related to the underlying muscularis mucosae.

Counts of Leu 1 positive lymphocytes in the surface epithelium are significantly lower than counts of T3 and Leu 9 positive lymphocytes in both controls $(p<0.01$ and $p<0.005)$ and coeliacs $(p<0.005$ and $p<0.01)$ whether expressed as counts per $\mathrm{mm}$ epithelium or muscularis mucosae. In coeliac crypt epithelium counts of Leu 1 positive lymphocytes per mm muscularis mucosae are significantly lower than T3 positive lymphocytes $(p<0 \cdot 01)$. The counts of Leu 5 positive lymphocytes are significantly lower than T3 positive lymphocytes in coeliac surface and crypt epithelium whether expressed per mm epithelium $(p<0.0005$ and $p<0.01)$ or mm muscularis mucosae $(\mathrm{p}<0.01$ and $\mathrm{p}<0.005)$. In the crypt epithelium there are smaller numbers of all lymphocyte populations, whether they are expressed as counts per $\mathrm{mm}$ epithelium or $\mathrm{mm}$ muscularis mucosae, than in the surface epithelium of both controls and coeliacs. In coeliac disease the numbers of intraepithelial Leu 1 and Leu 5 positive cells per mm of surface epithelium are significantly increased, but the changes in the crypt epithelium are not statistically significant and there are no significant changes in the counts in the surface intraepithelial lymphocytes per unit length of muscularis mucosae.

Of the T-lymphocyte subsets, Leu 2 positive cells predominate in both surface and crypt epithelium of controls and coeliacs. Leu 3 positive cells are present in small numbers. The Leu 2/Leu 3 ratio is approximately 8 in the surface epithelium and 5 in the crypt epithelium in controls and in coeliacs. The counts of both subsets in the surface epithelium are increased in coeliac disease when measured relative to the length of the surface epithelium but not when related to the underlying muscularis mucosae. In the crypt epithelium, for Leu 2 only, the situation is reversed and there is a significant increase in the counts related to the muscularis mucosae but not related to length of crypt epithelium.

The sum of the Leu 2 and Leu 3 bearing lymphocytes was computed and the differences between this and the T3 and Leu 9 positive lymphocyte counts is given. In the surface and crypt epithelium of controls the mean values of the sum of the Leu 2 and Leu 3 bearing cells and the T3 positive cells correspond, although there is a wide individual range. In coeliacs there is a marked difference 
between the populations in the surface epithelium, with many more lymphocytes expressing $\mathrm{T} 3$ than Leu 2 or Leu 3 markers although again there is a wide individual range. Similar results are obtained when Leu 9 is substituted for T3. There is also a significant increase in the gap between the counts of T3 positive and Leu 1 and Leu 5 positive lymphocytes per $\mathrm{mm}$ of surface epithelium in coeliacs compared with controls; in the crypt epithelium also the gap between counts of T3 positive and Leu 5 positive lymphocytes is significantly increased in coeliac patients when expressed as cells per $\mathrm{mm}$ muscularis mucosae.

In the lamina propria the counts obtained using the pan-T lymphocyte markers T3, Leu 1 and Leu 5 closely correspond in control subjects. Leu 9, however, marks a significantly smaller population (approximately $50 \%$ of all T-cell markers). There is a large population of Leu 3 positive lymphocytes as well as Leu 2 positive lymphocytes in both controls and coeliacs. The Leu 3/Leu 2 ratio is similar in both - approximately $0 \cdot 4$. In all lamina propria cell counts there is a wide range of individual variation both in controls and coeliacs but no significant differences are detected.

\section{Discussion}

The use of an immunocytochemical technique allows the in situ study of the lymphocytes in normal and abnormal small intestinal mucosa, and can provide valuable evidence of changes in the nature and perhaps the function of these cells in disease. The immunoperoxidase technique also allows the accurate localisation of the cells and correlation of the cell counts with structural changes such as villous atrophy and changes in mucosal volume. A monoclonal antibody reacts to a single epitope which may be used to define the operational set or subset to which the cell belongs and also may be related to the functional state of the cell. Some antibodies may react to an antigen which has a known specific function that is essential to the definition of a particular cell type. An example of this is the T3 antigen which is associated with the T-lymphocyte antigen receptor, and which is probably the best marker of a functionally mature peripheral $\mathrm{T}$ lymphocyte. The T4 (Leu 3) and T8 (Leu 2) antigens are also associated with antigen reception in $\mathrm{T}$ lymphocytes and appear to mark cells responding to antigen in association with HLA class I and II molecules respectively. Other antibodies such as the other 'pan-T-lymphocyte' markers do not have such a clear relation to physiological role, although Leu 5 is related to the $E$ receptor. This may be expressed by a proportion of natural killer (NK) cells as well as by functional T-lymphocytes. ${ }^{12}$ Leu 9 is also expressed by null cells as well as T-lymphocytes. Multiple markers can be used to define the lineage of the cells more precisely as a single marker also may be present on cells of different lineage - for example, Leu 3 antigen is present on cells of the monocyte/macrophage and Langerhan's lineage as well as a subset of T-lymphocytes. The use of multiple monoclonal antibodies allows the establishment of a detailed phenotypic pattern for the population of lymphocytes under study. Changes of this pattern in diseases may, as well as defining population changes, indicate changes in the functional state of the cells even if the numbers are unchanged. This can complement studies of functional immunology using peripheral blood or even extracted cells from the gut mucosa which can be criticised on the grounds that the cells are not representative of the specialised mucosal cells or are affected by the process of extraction.

Comparison of the counts of the cells demonstrated by the various 'pan-T-lymphocyte' antibodies in the intestinal epithelium shows unequivocally that the vast majority of cells are functional $T$ lymphocytes as judged by marker profile. There are no definite numbers of null cells or $E$ receptor positive, T3 negative lymphocytes or more than occasional cells carrying the Leu 7 or Leu 11 antigens which might form an important distinct NK cell population. This is consistent with previous studies $^{10} 15$ but the data also allow the question raised previously ${ }^{8}$ - whether intraepithelial lymphocytes are increased or decreased in coeliac disease to be phrased more precisely in terms of cell type, and offers a possible answer as to whether these cells could be playing an active role in the disease process such as has been suggested by previous morphological studies of intraepithelial lymphocytes, experimental studies of graft-versus-host reaction, ${ }^{6}$ and allograft rejection. ${ }^{1}$

Our study shows that within the surface epithelium of coeliacs the total number of T-lymphocytes per unit length, and hence per unit area, of underlying muscularis mucosae is not increased, although the relative density within the reduced epithelial compartment, expressed as counts per unit length of epithelium, is much higher. This result is consistent with the findings of previous studies ${ }^{7}$ of intraepithelial lymphocytes identified morphologically. There are, however, changes in the marker patterns in the intraepithelial lymphocytes which might suggest an altered functional state rather than just an increase in density because of the nonspecific crowding of cells into a reduced surface epithelial compartment. There is an increase in the 
density of Leu 2 positive lymphocytes but there is also in many cases a population of T3 lymphocytes which appear not to express Leu 2 or Leu 3 in amounts detected by immunocytochemical means. As well as an increase in the number of Leu 1 and of Leu 5 positive intraepithelial lymphocytes, there is also an increase in the relative density of $T$ lymphocytes which are Leu 1 or Leu 5 negative. The significance of these changes is supported by comparison of lymphocyte populations in the surface epithelium with those in the crypt epithelium and the lamina propria where there is close correspondence of the counts of all pan-T-lymphocyte markers. This suggests that the altered patterns of staining of intraepithelial lymphocytes is not an artefact due to low affinity of the antibody and insensitivity of the method. The possibility exists, however, that changes in the intraepithelial environment of the lymphocytes, rather than changes in the lymphocytes themselves, affect the observed staining pattern. A functional significance is suggested for these changes in coeliac disease, and in particular for the specific population of T3 positive, Leu 2 and Leu 3 negative, T-lymphocytes in the surface epithelium, from other studies. A study of T-cell subsets in Sjogren's disease, in which immune mechanisms at epithelial surfaces are also thought to be involved, has demonstrated a population of 'low antigen density Leu 2 positive cells' ${ }^{16}$ That study used flow cytometry which is a more sensitive method than immunocytochemistry, and it is possible that our apparently negative staining cells may correspond to this population. It was suggested that the 'low antigen density Leu 2 positive cells' represented an abnormal differentiation of the cytotoxic/suppressor lymphocytes, but it is also possible that this represents an activation change. There is support for this latter interpretation from studies of the T8 (Leu 2) antigen by Reinherz et $a^{13}$ which led them to suggest that this antigen might not be expressed by committed cytotoxic/suppressor clones with high affinity for 'neoantigen'. This interpretation is supported by previous morphological studies which have shown enlargement and increased mitotic activity of intraepithelial lymphocytes. The lymphocyte populations of the crypt epithelium appear to be different in number and composition from those of the surface epithelium. Intraepithelial lymphocytes were found in smaller numbers in the crypts than at the surface in both normals and coeliacs. The ratio of cells carrying Leu 3 and Leu 2 antigens - the 'helper/suppressor' ratio - is intermediate between that of the surface epithelium, where Leu 2 positive cells vastly predominate, and the lamina propria or peripheral blood. The crypt compartment of the epithelium expands in coeliac disease and there is a corresponding significant increase in the total counts of T-lymphocytes and Leu-2 positive cells, but there is no significant change in the density of these cells per unit length of crypt epithelium. This suggests a different lymphocyte-epithelial relationship in the crypts to that in the surface epithelium, both in normal and coeliac mucosa.

There has been much indirect evidence to suggest that cell mediated immunity is important in the pathogenesis of coeliac disease. The changes reported here in the marker patterns of lymphocytes in the surface epithelium provide new, and more direct, evidence that these cells are part of an active cell mediated immune response which may be involved in the pathogenesis of coeliac disease.

We thank Mr P M Stephenson for technical help and the Trent Regional Health Authority Research Committee for generous financial support.

\section{References}

1 Ferguson A, Parrott DMV. Histopathology and time course of rejection of allografts of mouse small intestine. Transplantation 1973; 15: 546-54.

2 Bullen A, Losowsky MS. Cell mediated immunity to gluten fraction III in adult coeliac disease. Gut 1978; 19: $126-31$.

3 Falchuk ZM, Strober W. Gluten-sensitive enteropathy: synthesis of anti-gliadin antibody in vitro. Gut 1974; 15: 947-52.

4 Scott BB, Goodall A, Stephenson P, Jenkins D. Small intestinal plasma cells in coeliac disease. Gut 1984; 25: 41-6.

5 O'Farrelly C, Feighery C, Greally JF, Weir DG. Cellular response to alpha-gliadin in untreated coeliac disease. Gut 1982; 23: 83-7.

6 Ferguson A, Ziegler K, Strobel S. Relevance of intestinal mucosal $\mathrm{T}$ cells to the immunology of coeliac disease and food allergy. In: Chadwick VS, ed. Mechanism of gastrointestinal inflammation. Welwyn: Smith Kline and French, 1984: 85-7.

7 Marsh MN. Functional and structural aspects of the epithelial lymphocyte, with implications for coeliac disease and tropical sprue. Scand J Gastroenterol 1985; 20: suppl 114: $55-75$.

8 Corazzo GR, Frazzoni M, Gasbarrini G. Jejunal intraepithelial lymphocytes in coeliac disease: are they increased or decreased? Gut 1984; 25: 158-62.

9 Selby WS, Janossy G, Goldstein G, Jewell DP. T lymphocyte subsets in normal intestinal mucosa - the distribution and relationship to MHC-derived antigens. Clin Exp Immunol 1981; 44: 453-8.

10 Selby WS, Janossy G, Bofill MA, Jewell DP. Lymphocyte subpopulations in the human small intestine. The findings in normal mucosa and in the mucosa of 
patients with adult coeliac disease. Clin Exp Immunol 1983; 52: 219-28.

11 Fakhri O, Hobbs JR. Detection of antibodies which can cooperate with lymphocytes. Lancet $1972 ; 2:$ 403-6.

12 Beverley PCL. Hybridomas, monoclonal cells and analysis of the immune system. Br Med Bull 1984; 40: 213-7.

13 Reinherz EL, Meuer SC, Schlossman SF. The delineation of antigen receptors on human $\mathrm{T}$ lymphocytes. In: Inglis JR, ed. $T$ lymphocytes today. Amsterdam: Elsevier, 1983: 26-9.
14 Scott BB, Jenkins D. Endoscopic small intestinal biopsy. Gastrointestinal endoscopy 1981; 27: 162-7.

15 Cerf-Bensussan N, Guy-Grand D, Griscelli C. Intraepithelial lymphocytes of human gut: isolation, characterisation and study of natural killer activity. Gut 1985; 26: 81-8.

16 Bakhshi A, Miyasaka N, Kavathas P et al. Lymphocyte subsets in Sjogren's syndrome: a quantitative analysis using monoclonal antibodies and the fluorescenceactivated cell sorter. J Clin Lab Immunol 1983; 10: 63-9. 